\title{
Observations of Neutron-Capture Elements in the Early Galaxy
}

\author{
C. Sneden ${ }^{\mathrm{a}} *$, J. J. Cowan ${ }^{\mathrm{b}},{ }^{\dagger}$, and J. E. Lawler ${ }^{\ddagger}$ \\ aDepartment of Astronomy and McDonald Observatory, University of Texas, Austin, \\ TX 78712, USA
}

bepartment of Physics and Astronomy, University of Oklahoma, Norman, OK 73019, USA

${ }^{\text {c} D e p a r t m e n t ~ o f ~ P h y s i c s, ~ U n i v e r s i t y ~ o f ~ W i s c o n s i n, ~ M a d i s o n, ~ W I ~} 53706$ USA

Neutron-capture elements in low metallicity Galactic halo stars vary widely both in overall contents and detailed abundance patterns. This review discusses recent observational results on the n-capture elements, discussing the implications for early Galactic nucleosynthesis of: (a) the star-to-star "bulk" variations in the n-capture/Fe abundance ratios; (b) the distinct signature of rapid n-capture synthesis events in many (most?) of the lowest metallicity stars; (c) the existence of metal-poor stars heavily enriched in the products of slow n-capture synthesis reactions; and (d) the now-routine detection of radioactive thorium (and even uranium in one and possibly two cases) in the spectra of metal-poor stars.

\section{NEUTRON-CAPTURE ELEMENTS}

Neutron-capture elements are those heavy elements whose isotopes are primarily synthesized in neutron bombardment reactions; these include all elements with $\mathrm{Z}>30$. The relative rates of neutron ingestion by nuclei compete with $\beta$-decay rates to determine the final abundance distribution. If the neutron flux is small enough that all unstable nuclei may undergo $\beta$-decay between successive neutron captures, element synthesis proceeds up the valley of $\beta$ stability. This slow neutron addition is called the $s$-process, and occurs principally in the He-burning zones of low and intermediate mass AGB stars. If however the neutron flux is very large compared to $\beta$-decay rates, extremely neutron-rich nuclei are created which then rapidly undergo $\beta$-decay back toward stability. This rapid neutroncapture ( $n$-capture) synthesis, called the $r$-process, can occur in several situations during supernova deaths of high-mass stars and their creation of neutron-star remnants.

Isotopes of the $n$-capture elements may be created by the $s$-process, the $r$-process, or both. The solar-system abundances of these isotopes have been built in roughly equal measure of products of the $r$ - and $s$-process. The manner in which isotopic (and hence

\footnotetext{
*The authors all thank our colleagues who have contributed to the results presented here. Research supported by U.S. NSF grant AST-9987162.

${ }^{\dagger}$ Research supported by U.S. NSF grant AST-9986974.

${ }^{\ddagger}$ Research supported by U.S. NSF grant AST-981940.
} 
elemental) abundance fractions are usually determined [1, 2, 3] deserves comment here. In the $s$-process the product of isotopic abundance and neutron-capture cross-section is a well-defined slowly varying function of atomic mass, which can be matched to the observed (meteoritic) abundances of isotopes that only can be made in the $s$-process. The $s$-process abundances of other isotopes can then be predicted from this match. Then the $r$-process abundances are either taken directly from meteoritic data for $r$-only isotopes, or computed by subtraction of the $s$-process amounts from the total abundances for those isotopes that can be synthesized in both $n$-capture processes. Finally, since only total elemental abundances may normally be determined from stellar spectra, the isotopic $r$ and $s$-process isotopic abundances for each element are summed.

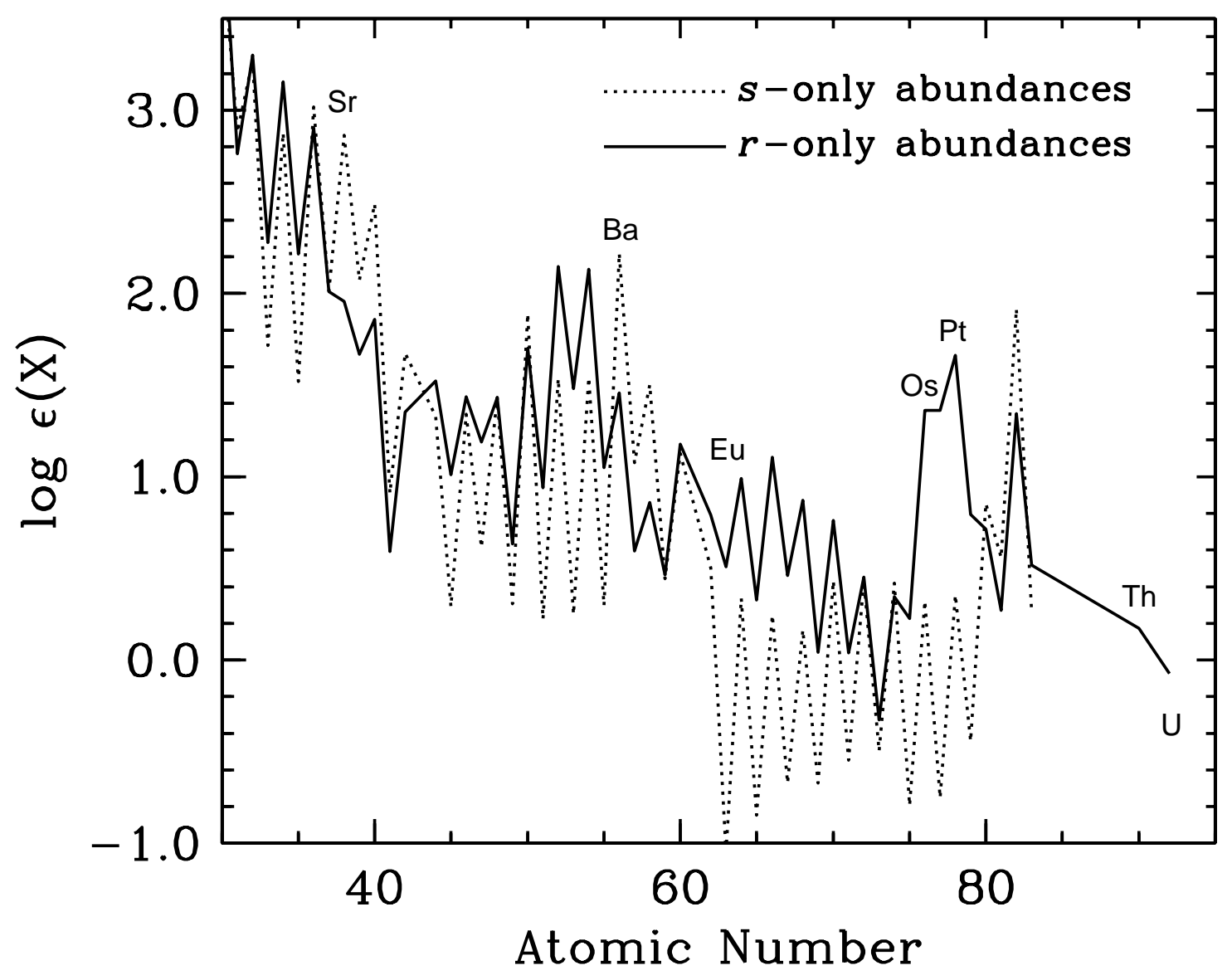

Figure 1. Abundance distributions of $n$-capture elements in the solar system, separated into their $r$-process-only and $s$-process-only components [ [3], as functions of atomic number Z. Abundances are given in standard stellar spectroscopic notation: $\log \epsilon(\mathrm{A}) \equiv$ $\log _{10}\left(\mathrm{~N}_{\mathrm{A}} / \mathrm{N}_{\mathrm{H}}\right)+12.0$.

A recent re-assessment [ [3] of the solar-system elemental abundances is shown in Figure [1. With this figure a caution must be given. For those elements whose solar-system 
abundances are dominated by the $s$-process (e.g. Sr, Ba, La), the small $r$-process component obtained in the subtraction process carries with it the abundance uncertainty estimate of the $s$-process component, and this value can in extreme cases be as large as the inferred $r$-process abundance. Thus the solar-system $r$-process abundance set should be treated with some caution. But for the foreseeable future this distribution will remain the standard, because direct predictions of $r$-process abundances (e.g., [ [4, 5]) must rely on mass models of extremely neutron-rich nuclei, almost none of which has ever been experimentally detected. Theoretical assessments of the solar-system $s$ - and $r$-process abundances have also been made (e.g. [ 6]), and these yield similar results to those displayed in Figure 1).

\section{NEUTRON-CAPTURE ELEMENTS AT LOW METALLICITY}

A dominant aspect of $n$-capture element abundances in very metal-poor halo stars is their extremely large variability "in bulk" with respect to the Fe-peak elements (for early indications compare the abundances reported for HD 122563 [7] with those of HD 115444 [ 8], and for larger-sample surveys see, e.g., [ 9, 10, 11, 12]). The observed star-to-star range in $[<n$-capture $>/ \mathrm{Fe}]$ grows with decreasing metallicity, exceeding observational errors at $[\mathrm{Fe} / \mathrm{H}] \approx-2.0$ and reaching a scatter factor of more than a thousand below $[\mathrm{Fe} / \mathrm{H}] \approx-2.5$ (e.g., Figure 12 of [ [12]). The lowest metallicity stars are usually faint and high resolution spectra of them requires the use of $8 \mathrm{~m}$-class telescopes.

Proof of the star-to-star $n$-capture scatter can be had through simple spectrum comparisons of relatively bright stars, as has been done by [ 3], whose Figure 3 is adapted as our Figure 2. The two stars shown here have almost identical atmospheric parameters, and the spectral region of the top figure panel suggests that their metallicities and light element abundance ratios are about the same. But the $n$-capture element features have strengths that differ by nearly a factor of ten, and it is easy to show that derived abundances of these elements differ by the same amount with little dependence on atmospheric modeling uncertainties. And the large star-to-star scatter can probably have only one interpretation: the influence of individual and localized nucleosynthesis events in short-lived stars in a poorly-mixed early Galactic halo.

A second feature of $n$-capture elements in metal-poor stars is the general trend toward pure $r$-process signatures (at least for elements with $\mathrm{Z} \geq 56$ ) at lowest metallicities. Significant exceptions to this trend are known to exist. In particular there are some stars with $[\mathrm{Fe} / \mathrm{H}]<-2.0$ with large overabundances of $\mathrm{C}$ and $s$-process-dominated elements such as $\mathrm{Sr}, \mathrm{Ba}$ and even $\mathrm{Pb}$ (e.g. [ 13, 14]). Such objects may have received these enhanced abundances via mass transfer from a former AGB companion star. A more thorough discussion of these important stars has been given by S. G. Ryan at this conference, and will not be commented upon further here.

For more "normal" low metallicity stars, a downward trend in [Ba/Eu] is observed with decreasing metallicity (the pioneering observational and theoretical work here were done by [15, 16], and for a recent summary see Figure 3 of [ 17] and Figure 7 of [ 3]). This decrease, which tends toward $[\mathrm{Ba} / \mathrm{Eu}] \approx-1.0$ below $[\mathrm{Fe} / \mathrm{H}] \approx-2.5$, is qualitatively and quantitatively in agreement with essentially complete absence of products of $s$-process synthesis in the most metal-poor stars. The trend combined with the large star-to-star 
scatter in the bulk amounts of the $n$-capture elements noted above rounds out the suggested picture of $r$-process synthesis occurring in and around the supernova deaths of high mass, very low metallicity stars, and seeding their local ISM out of which quickly formed the metal-poor stars that we observe today.

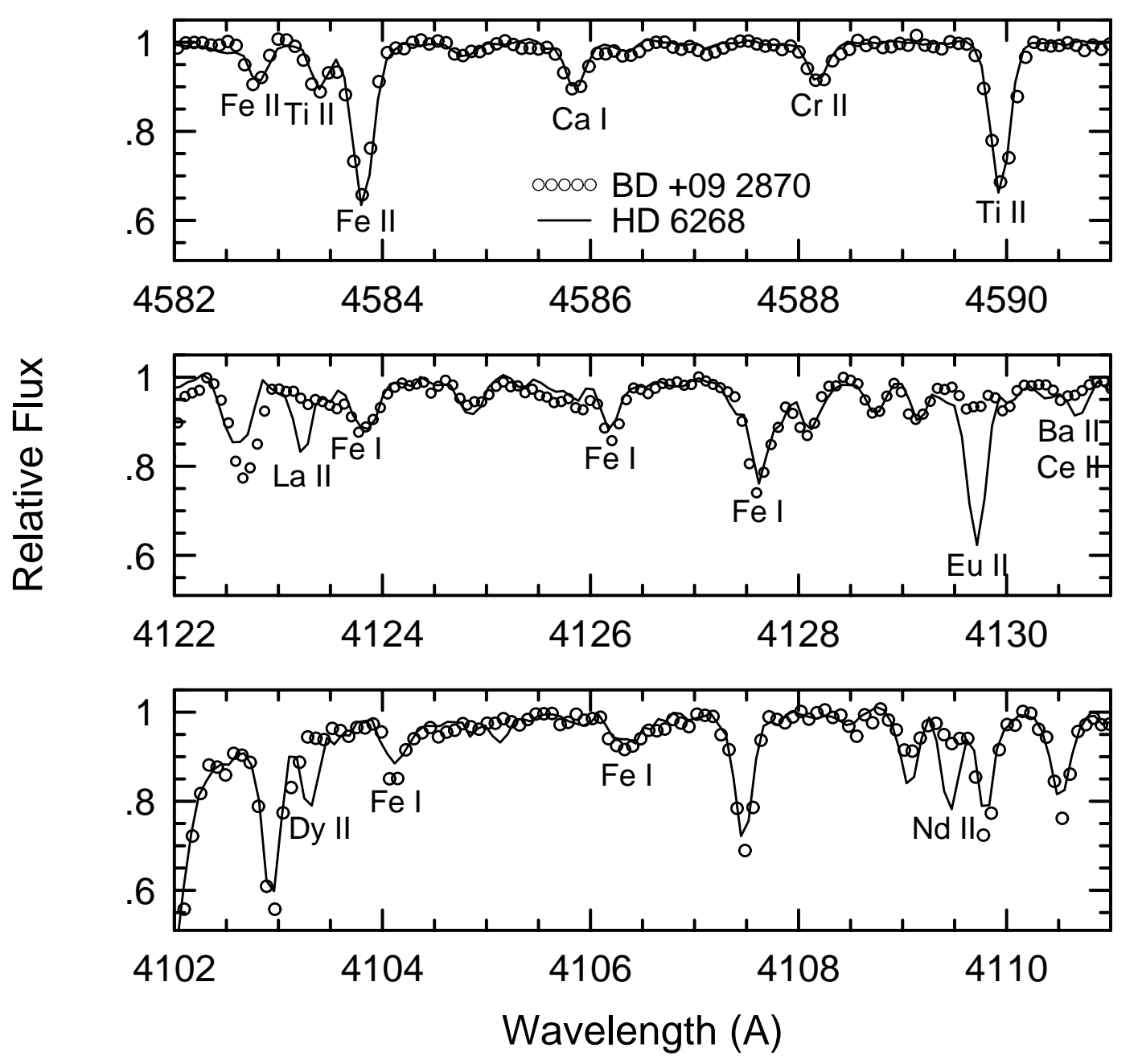

Figure 2. Comparison of the spectra of two metal-poor giant stars in a wavelength region dominated by Fe-peak species features (top panel), and in two regions with significant $n$-capture features. This figure is adapted from Figure 3 of [ [3]. These two stars have very similar atmospheric parameters and metallicities: $\mathrm{T}_{\text {eff }}=4650 \mathrm{~K}, \log \mathrm{g}=1.50, \mathrm{v}_{t}=$ $1.7 \mathrm{~km} \mathrm{~s}^{-1},[\mathrm{Fe} / \mathrm{H}]=-2.4$.

Unfortunately another undeniable aspect of the $[\mathrm{Ba} / \mathrm{Eu}]$ trend with metallicity is its large scatter at any $[\mathrm{Fe} / \mathrm{H}]$ value. It is possible (even likely) that some of the scatter is intrinsic to the stars, caused by $s$-processing even in the early Galaxy. Some of the very metal-poor stars with $[\mathrm{Ba} / \mathrm{Eu}]$ ratios much greater than the $r$-process value probably have undergone some a transfer episode from a higher mass companion which is now 
a compact object. But a lot of the $[\mathrm{Ba} / \mathrm{Eu}]$ scatter must be due to observational and analytical uncertainties, particularly in the derivation of Ba abundances. Usually just the very few strong Ba II low-excitation lines are available for analysis, and these features are saturated in cool giants that are most often studied at low metallicities.

There are two approaches that can mitigate this problem. First, Ba abundances should be determined for main sequence stars, as has been recently done in [ 12]. The Ba II lines are intrinsically much weaker in upper main sequence stars than they are in giants of similar metallicity. The problem with this approach lies in simultaneous detection of Eu II features, which become almost undetectably weak in very low metallicity main sequence stars. Second, instead of using Ba II lines, a switch could profitably be made to analyses of La II (which is also predominantly an $s$-process product in solar-system material). This species offers several analytical advantages: (a) La has just one stable isotope, compared with five abundant ones for Ba; (b) La II lines are more numerous and weaker than Ba II lines, and usually are of comparable strengths to those of Eu II; and (c) accurate transition probabilities and hyperfine structure parameters are known for many La II lines [ 18]. Preliminary results of a new large-sample study of [La/Eu] have been given in [19], and they show a smaller star-to-star scatter than does [Ba/Eu] and the same trend to a pure $r$-process ratio at lowest metallicities. The use of La II instead of $\mathrm{Ba}$ II lines is recommended for judging the $s$-process contents of all stars where the $n$-capture features all are strong.

\section{DETAILED ABUNDANCE DISTRIBUTIONS IN $r$-PROCESS-RICH STARS}

A few very metal-poor stars have been discovered to have both very large overall $n$-capture element contents (here arbitrarily defined as $[\mathrm{Eu} / \mathrm{Fe}] \sim+1$ or greater) and nearly pure $r$-process signatures among these elements. Such stars include HD 115444 $([\mathrm{Fe} / \mathrm{H}]=-2.9,[\mathrm{Eu} / \mathrm{Fe}]=+0.9[20]), \mathrm{CS} 22892-052([\mathrm{Fe} / \mathrm{H}]=-3.1,[\mathrm{Eu} / \mathrm{Fe}]=+1.6[21])$, $\mathrm{BD}+173248([\mathrm{Fe} / \mathrm{H}]=-2.1,[\mathrm{Eu} / \mathrm{Fe}]=+0.9[22])$, and CS 31082-001 $([\mathrm{Fe} / \mathrm{H}]=-2.9$, $[\mathrm{Eu} / \mathrm{Fe}]=+1.6[23)$. Such stars are excellent laboratories for the study of $n$-capture elements because their normally dominant Fe-peak transitions are very weak in these low metallicity stars, and the relative overabundances of the $n$-capture elements reveals many spectral features that are normally hidden in stellar spectra. For example, Tb II lines are much more readily identified in the spectrum of CS 22892-052 than they are in the solar spectrum [24].

In Figure 3 we summarize the current [25] CS 22892-052 n-capture elemental abundance set, comparing it to the scaled solar-system abundance distribution [3]. The abundance matches shown here lead to the following brief comments, reemphasizing some conclusions from our previous contributions.

First, for the 17 observed stable elements with $\mathrm{Z} \geq 56$, a near-perfect match exists with the scaled solar-system $r$-process curve. The fit is good enough that elements which either deviate from the solar curve (e.g. Er) or have large line-to-line scatter (e.g. Nd) point to areas for further study in nuclear and atomic physics, respectively. Comparing the abundances shown here to those of the first large abundance study of CS 22892-052 [26], the major sharpening of the newer abundance results (smaller line-to-line scatters) may be traced to several recent extensive lab studies of rare-earth elements (for reviews and 
comments on recent work in this area see [27, 28]). Note finally that the excellent agreement with scaled solar system abundances now has been extended to isotopic abundances for $\mathrm{Eu}$ in three $r$-process-rich stars [29].

Second, elements of the third $n$-capture peak $(76 \leq \mathrm{Z} \leq 82)$ have their transitions mainly below $3500 \AA$, and thus some of these abundances have been obtained from HST STIS observations. It is more difficult to derive reliable abundances in the UV because even very metal-poor giants have rich atomic and molecular line spectra in this wavelength regime. Even so, the abundances of this element group are consistent with the same scaled solar curve that matches the abundances of the rare-earth elements.

Third, the long-lived radioactive element Th is detected in CS 22892-052 and indeed it has been observed in many metal-poor giants [ 30, 31]. The derived Th abundance, when ratioed to the abundance of a stable $n$-capture element (usually taken to be Eu) can lead to age estimates for the oldest stars of the Galaxy. The prospects and problems of such nucleocosmochronology have been discussed in several papers (e.g. [ 5, 32, 33]) and space does not permit further discussion here. An exciting new development has been the detection of U, a second chronometer element, in CS 31082-001 [23] and its ratio with respect to Th indicates a decay age of about $15 \mathrm{Gyr}$, in agreement with other kinds of Galactic age estimates. See also [22] for an additional tentative detection of $U$ in a metal-poor halo star and a similar radioactive age estimate.

Fourth, lighter $n$-capture elements, those with $38 \leq \mathrm{Z} \leq 50$, show some deviations from the scaled solar $r$-process distribution, particularly for $\mathrm{Y}, \mathrm{Mo}, \mathrm{Pd}$, and $\mathrm{Ag}$. One caution must be given here, that for most of these elements the number of lines participating in the derived abundance is significantly smaller than the number available for abundance determinations of rare earth elements; hence the generally larger error estimates that we attach to these points. Suggestions that the $r$-process nucleosynthetic history of the lighter $n$-capture elements may be complex began with [ 34] and has no clear resolution today. The deviations may signal the presence of a second type of $r$-process as proposed in [35]. Alternatively, models have been suggested that can synthesize the whole range of these observed abundances in one site (e.g. [36])

Fifth, the lightest elements attributed mostly to $n$-capture processes cannot be detected on our CS 22892-052 spectra, and the derived upper limits to their abundances are more than an order of magnitude below the scaled solar $r$-process curve. Clearly these two elements either owe their origin to a different nucleosynthetic process or the $r$-process simply may have pushed beyond these elements.

\section{SUMMARY}

A rich variety of $n$-capture abundance distributions has been found in low metallicity Galactic halo stars, and discoveries of new stars with unique chemical composition mixes show no signs of abating. Early Galactic nucleosynthesis has littered the halo with many clues, and it will take the combined efforts of astronomers working with halo low resolution surveys, stellar spectroscopists, lab and theoretical atomic physicists, experimental and theoretical nuclear physicists, and supernova modelers to properly decode the messages contained in the $n$-capture abundances of metal-poor stars. 


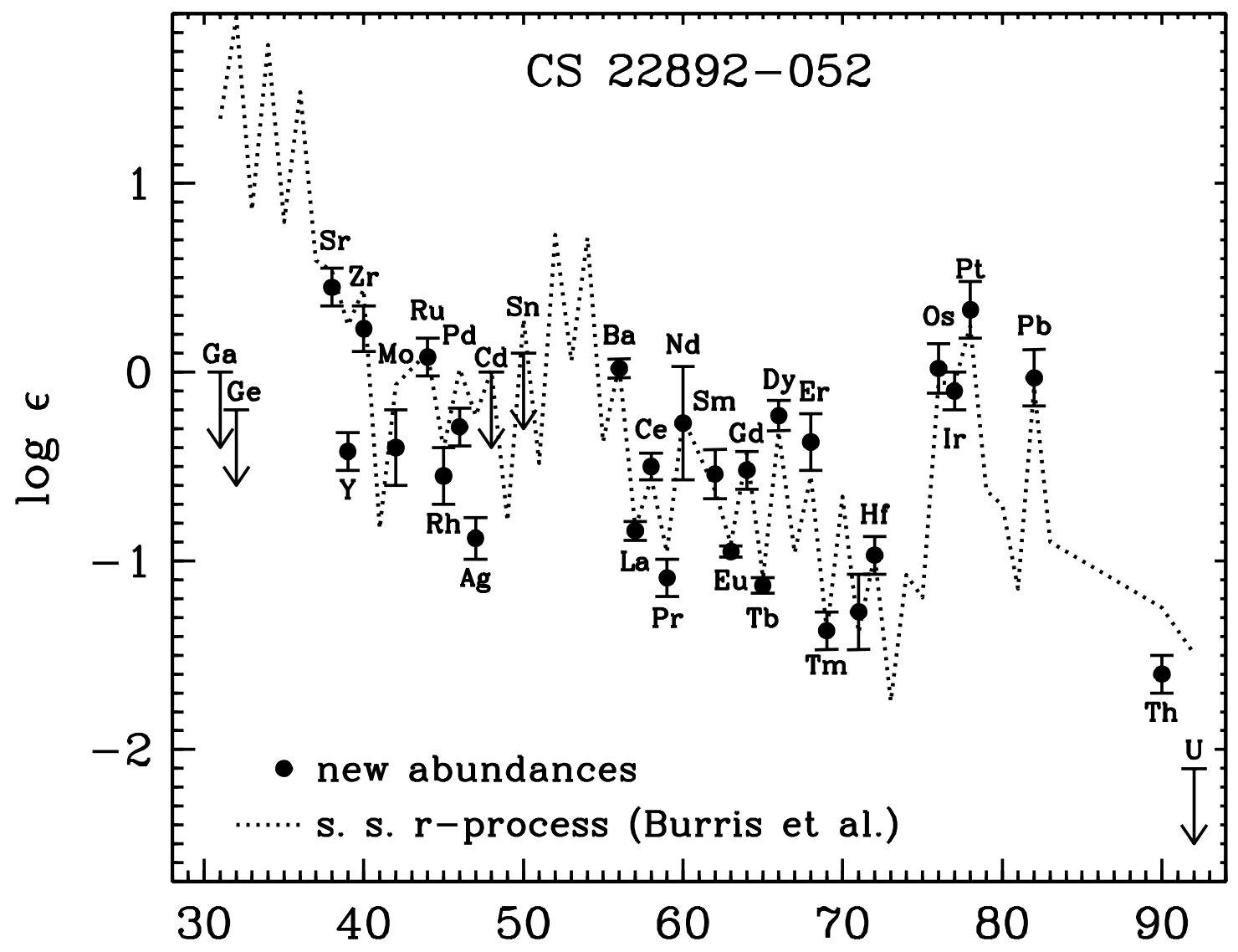

Figure 3. Observed $n$-capture abundances [25] in the halo giant star CS 22892-052 compared to the $r$-process solar-system abundance distribution of Figure 1, scaled to match the observed Eu abundance. The error bars on the observed points represent the sample standard deviations of the abundances of each element.

\section{REFERENCES}

1. A. G. W. Cameron, Ap. Sp. Sci., 82 (1982) 123.

2. F. Käppeler, H. Beer, and K. Wisshak, Rep. Prog. Phys., 52 (1989) 945.

3. D. L. Burris, C. A. Pilachowski, T. L. Armandroff, C. Sneden, J. J. Cowan, and H. Roe, Ap. J., 544 (2000) 302

4. S. Goriely and M. Arnould, Astr. Ap., 322 (1997) L29.

5. J. J. Cowan, B. Pfeiffer, K.-L. Kratz, F.-K. Thielemann, C. Sneden, S. Burles, D. Tytler, and T. C. Beers, Ap. J., 521 (1999) 194.

6. C. Arlandini, F. Käppeler, K. Wisshak, R. Gallino, M. Lugaro, M. Busso, and O. Staniero, Ap. J. 525 (1999) 886

7. G. Wallerstein, J. L. Greenstein, R. Parker, H. L. Helfer, and L. H. Aller, Ap. J., 137 (1963) 280.

8. R. Griffin, R. Griffin, B. Gustafsson, and T. Vieira, Mon. Not. Roy. Astr. Soc. 198 (1982) 637. 
9. K. K. Gilroy, C. Sneden, C. A. Pilachowski, and J. J. Cowan, Ap. J. 327 (1988) 298. 10. A. McWilliam, G. W. Preston, C. Sneden, and S. Shectman, Astr. J. 109 (1995) 2736. 11. S. G. Ryan, J. E. Norris, and T. C. Beers, Ap. J. 471 (1996) 254.

12. E. Carretta, R. Gratton, J. G. Cohen, T. C. Beers, and N. Christlieb, Astr. J., 124 (2002) 481.

13. S. Van Eck, S. Goriely, A. Jorissen, and B. Plez, Nature, 412 (2001) 793.

14. W. Aoki, J. E. Norris, S. G. Ryan, T. C. Beers, and H. Ando, Ap. J., 576 (2002) L141.

15. M. Spite and F. Spite, Astr. Ap. 67 (1978) 23.

16. J. W. Truran, Astr. Ap., 97 (1981) 391

17. J. J. Cowan, C. Sneden, and J. W. Truran, in Relativistic Astrophysics: $20^{\text {th }}$ Texas Symposium, eds. J. C. Wheeler and H. Martel (2001) 337.

18. J. E. Lawler, G. Bonvallet, and C. Sneden, ApJ, 556 (2001) 452.

19. J. Simmerer and C. Sneden, Bull. Am. Astr. Soc., 33 (2002) 1512

20. J. Westin, C. Sneden, B. Gustafsson, and J. J. Cowan, Ap. J., 530 (2000) 783

21. C. Sneden, J. J. Cowan, I. I. Ivans, G. Fuller, S. Burles, T. C. Beers, and J. E. Lawler, Ap. J., 533 (2000) L139.

22. J. J. Cowan et al., Ap. J., 572 (2002) 861

23. V. Hill et al., Astr. Ap., 387 (2002) 560

24. J. E. Lawler, M. E. Wickliffe, C. R. Cowley, and C. Sneden, Ap. J. Supp., 137 (2001) 341.

25. C. Sneden et al., Ap. J., to be submitted (2002).

26. C. Sneden, A. McWilliam, G. W. Preston, J. J. Cowan, D. L. Burris, and B. J. Armosky, Ap. J., 467 (1996) 819

27. G. M. Wahlgren, Phys. Scr., T100 (2002) 22.

28. C. Sneden, J. E. Lawler, and J. J. Cowan, Phys. Scr., T100 (2002) 22.

29. C. Sneden, J. J. Cowan, J. E. Lawler, S. Burles, T. C. Beeers, and G. M. Fuller, Ap. J., 566 (2002) L25.

30. P. François, M. Spite, and F. Spite, Astr. Ap., 274 (1993) 821

31. J. A. Johnson and M. Bolte, Ap. J., 554 (2001) 888.

32. S. Goriely and M. Arnould, Astr. Ap., 379 (2001) 1113.

33. H. Schatz, R. Toenjes, B. Pfeiffer, T. C. Beers, J. J. Cowan, and K.-L. Kratz, Ap. J., (2002) in press.

34. G. J. Wasserburg, M. Busso, and R. Gallino, Ap. J., 466 (1996) 109.

35. Y.-Z. Qian and G. J. Wasserburg, Phys. Rep., 333 (2000), 77.

36. A. G. W. Cameron, Ap. J., 562 (2001) 456. 